\title{
Effect of RH, Temperature, Light, and Plant Age on Infection of Lowbush Blueberry by Sphaerulina vaccinii
}

\author{
Pervaiz A. Abbasi, ${ }^{\dagger}$ Paul D. Hildebrand, Shawkat Ali, Debra L. Moreau, and Willy E. Renderos \\ Kentville Research and Development Centre, Agriculture and Agri-Food Canada, Kentville, Nova Scotia B4N 1J5, Canada
}

\begin{abstract}
Leaf spot and stem canker caused by Sphaerulina vaccinii is associated with premature defoliation in lowbush blueberry resulting in reduced yields. In this study, we investigated the impact of free water, $\mathrm{RH}$, temperature, light, and plant age on leaf infection under controlled conditions. On potato dextrose agar, germination of conidia was usually polar. Growth was minimal at 5 and $10^{\circ} \mathrm{C}$, increased at 15 and $20^{\circ} \mathrm{C}$, was maximal at $25^{\circ} \mathrm{C}$ and decreased at $30^{\circ} \mathrm{C}$. Percentage of germinated conidia on inoculated blueberry leaves incubated in dark controlled-humidity chambers for 3 days $\left(25^{\circ} \mathrm{C}\right)$ was $86.0,90.5$, 81.3 , and 28.3 in free water, $100,97.5$ or $95 \%$ RH, respectively. Germination did not occur at 90 or $85 \%$ RH. Infection of inoculated plants, however, was not favored by free water, but rather by high RH ( $>95 \%)$ and a $14-\mathrm{h}$ photoperiod $\left(180 \mu \mathrm{mol} / \mathrm{m}^{2}\right.$ per second). Infection failed in continuous darkness, continuous light, or continuous

proximity to a stomate, a penetration hypha formed at $\sim 90^{\circ}$ angles to the germ tube and took the closest path to the stomate. Stomatal penetration was usually direct, but occasionally appressorium-like hyphal swellings formed over stomatal openings. When inoculated plants were exposed to high $\mathrm{RH}(>95 \%)$ at various temperatures, infection occurred after 4 days at $10^{\circ} \mathrm{C}$, after 3 days at $15^{\circ} \mathrm{C}$ and after 1 day at 20 and $25^{\circ} \mathrm{C}$. Infection failed to occur at $30^{\circ} \mathrm{C}$. Disease severity also increased with duration of the humid period. When leaves were examined microscopically, those that had been incubated for 6 days showed a substantially greater network of epiphytic growth with more stomatal penetrations compared with those incubated for 3 days. Infection was substantially reduced when the humid period was interrupted by alternating days of low RH (60\%). Two-week-old leaves were 2.7 times more susceptible than 8 -week-old leaves.
\end{abstract} darkness followed by 4,8 , or $12 \mathrm{~h}$ of light. Light and scanning electron microscopy showed that hyphal penetration into stomata on abaxial leaf surfaces was strongly tropic. When germ tubes grew in close
Keywords: defoliation, environmental conditions, foliar disease, leaf spotting, wild blueberry
Lowbush blueberries or wild blueberries (Vaccinium angustifolium Ait. and V. myrtilloides Michx.) are native to Eastern North America and are one of the most valuable wild resource managed horticultural crops in Canada (Vander Kloet 1978). The berries are valued for their antioxidant content and other health benefits (De PascualTeresa et al. 2010; Forney and Kalt 2011). Canada is the world's largest producer of lowbush blueberries with most of the commercial production occurring in Quebec and the Atlantic provinces. Plants grow in clonal patches by underground rhizomes creating solid stands in fields (Bell et al. 2010; Vander Kloet 1978). The crop is produced on a 2-year production cycle. Fruits are harvested in August and then stems are pruned to ground level in late fall or early spring. In early June, new sprout stems emerge in the pruned fields and continue to elongate apically until early August. Flower buds develop on the new stems during late summer and autumn. After winter, foliage begins to expand during May and flowering occurs in June with harvest to follow. Pruning in alternate years promotes higher yields on straight stems and aids in mechanical harvesting.

The lowbush blueberry crop is susceptible to a number of fungal diseases throughout the production cycle that affect the foliage and fruit reducing yield and quality (Annis and Stubbs 2004; Drummond et al. 2009; Hildebrand et al. 2016; Percival and Dawson 2009; Yarborough et al. 2017). One such disease of importance is Sphaerulina leaf spot

${ }^{\dagger}$ Corresponding author: P. A. Abbasi; pervaiz.abbasi@agr.gc.ca

P. D. Hindebrand and W. E. Renderos are retired.

Funding: This work was supported by Agriculture and Agri-Food Canada and the Wild Blueberry Producers Association of Nova Scotia.

The author(s) declare no conflict of interest.

Accepted for publication 6 August 2021

(C) 2022 Her Majesty the Queen in Right of Canada as represented by the Minister of Agriculture and Agri-Food and stem canker caused by Sphaerulina vaccinii Ali, Hildebrand \& Abbasi (Mycosphaerellaceae, Dothideomycetes; Ali et al. 2021). This disease was previously known as Septoria leaf spot and stem canker based on the incomplete characterization of the causal fungus that appeared to be morphologically and genetically related to the Septoria/Mycosphaerella group of fungi (Hildebrand et al. 2010b). However, in 2021, the fungus was subjected to a more in-depth molecular analysis and found to be a new species, $S$. vaccinii (Ali et al. 2021). Symptoms of the disease have been described in the literature (Hildebrand and Renderos 2017; Hildebrand et al. 2010b, 2016). In Atlantic Canada, infection of young leaves and stem tissues occurs during June, and by late June or early July, initial symptoms appear on the undersurface of leaves as minute water-soaked spots. The spots increase in size, coalesce, and penetrate to the upper surface where they appear as irregular, reddish brown spots and cause leaves to drop usually beginning in mid-to-late July. Infections also occur on emerging stems in the sprout phase of production, but remain latent until spring of the following year. The symptoms on stems initially appear as reddish-purple spots that later enlarge, become slightly sunken, turn brown and produce a few pycnidia, but the stems are usually not killed. Conidia from pycnidia on stems and fallen overwintered leaves from the previous sprout season are the source of rain-splashed inoculum onto newly emerged foliage of the fruiting season. Inoculum production occurs for $\sim 4$ to 5 weeks beginning in late May, which coincides with much of the flowering period. Because most of the foliage on fruiting stems develops simultaneously, leaf infections occur more or less uniformly throughout the canopy. If infection is severe, premature leaf drop before harvest may occur resulting in reduced yields (Ali et al. 2021; Hildebrand et al. 2016).

A leaf spot disease also affects highbush (Vaccinium corymbosum L.) and rabbiteye (Vaccinium ashei Reade) blueberry caused by Septoria albopunctata Cooke. It differs from the lowbush blueberry pathogen in that it produces larger conidia in pycnidia within well-defined spots on current season leaves (Scherm 2017). It is widely distributed in the southeastern United States and is one of the most common foliar diseases on rabbiteye blueberry (Demaree and Wilcox 1947; 
Scherm and Krewer 2003, 2008; Scherm et al. 2003). It reduces photosynthesis of affected leaves (Roloff et al. 2004) and leads to premature defoliation in the autumn resulting in reduced flower bud production for the following year's crop (Ojiambo and Scherm 2005; Ojiambo et al. 2006; Williamson and Miller 2002). However, S. albopunctata has not been reported in Canada or Maine where lowbush blueberry is grown.

The practice of burn pruning lowbush blueberry fields in the fall or spring can control Sphaerulina leaf spot and stem canker, but due to the high cost of fuel for tractor-drawn burners, growers have opted for the less costly management practice of flail mowing, but this does not destroy infected plant debris (Hildebrand et al. 2010a). Accordingly, as of this writing, the disease is being controlled by fungicides applied during the bloom period when inoculum is present. While fungicides are effective, there is no information on environmental factors affecting infection by $S$. vaccinii with which to possibly time fungicide applications more precisely in relation to infection periods. The objectives of this study, therefore, were to explore the effect of leaf wetness, $\mathrm{RH}$, temperature, light, and plant age on infection of lowbush blueberry under controlled conditions.

\section{Materials and Methods}

Inoculum. A single conidial isolate of $S$. vaccinii (NS-01 = DAOMC 252344) was obtained from an overwintered lowbush blueberry leaf as described in Ali et al. (2021). In preliminary tests, this isolate was most aggressive among numerous ones tested. For longterm storage, small agar cubes of potato dextrose agar (PDA) with mycelial growth were immersed in sterile $15 \%$ dimethyl sulfoxide and kept at $-80^{\circ} \mathrm{C}$ and subcultured on PDA as required. To prepare inoculum, agar cubes were recovered from the freezer and placed in a large drop of sterile distilled water on a Petri dish and crushed. Several loopfuls of the resulting suspension were then thoroughly streaked onto PDA and allowed to grow for 3 to 5 days at $20^{\circ} \mathrm{C}$ on the laboratory bench. Conidia were gently washed from the agar with sterile distilled water and diluted to the desired concentration with aid of a hemocytometer.

Plant material and incubation. Two-year-old potted plants of $V$. angustifolium from seed of a controlled cross (Blomidon $\times$ Brunswick) were used for all experiments. A preliminary inoculation of potted blueberry plants indicated this cross was susceptible to Sphaerulina leaf spot and stem canker (Ali et al. 2021). The plants were obtained from a local nursery in late summer and kept outdoors until early winter when they were transferred to a dark cold storage at $1^{\circ} \mathrm{C}$ (Hildebrand and Braun 1991). Plants were retrieved when needed during March to August, fertilized once with 15-15-18 (N-P-K) at 2 g/liter (40 ml per plant) and allowed to leaf out in a greenhouse at 20 to $26^{\circ} \mathrm{C}$ with a $16-\mathrm{h}$ photoperiod supplemented with artificial lighting as required. Plants were inoculated when leaves were $\sim 2$ - to 3-weeks-old unless otherwise noted.

Our previous study showed that infection occurred through stomata (Ali et al. 2021) and so to standardize inoculation in relation to stomatal opening, plants were transferred from the greenhouse to a growth chamber $\left(25^{\circ} \mathrm{C}\right)$ where they were photo-entrained for $72 \mathrm{~h}$ with a 14-h photoperiod beginning at 09:00 $\mathrm{h}$. The abaxial surface of leaves was spray-inoculated to incipient run-off at the beginning of a photoperiod.

Inoculated plants were incubated in Plexiglas chambers $(98 \times 51 \times$ $61 \mathrm{~cm}$ ) situated in growth cabinets. Each incubation chamber was equipped with two DeVilbiss nebulizers (model 645; Devilbiss Healthcare, Somerset, PA) connected to a distilled water reservoir. When required, the air pressure of each was adjusted to provide a fog that kept the leaves wet but not to the extent of large droplet accumulation. When high $\mathrm{RH}>95 \%$ was required without free water on the foliage, a horticultural capillary mat on the floor of each chamber was kept saturated with water, but the nebulizers were turned off. RH was periodically checked with a Watchdog $\mathrm{RH}$ and temperature sensor (Spectrum Technologies Inc., Aurora, IL). Leaves were also examined by touch to verify water had not condensed on the foliage. When dry, low RH conditions were required, plants were incubated in a growth chamber set at $60 \pm 3 \% \mathrm{RH}$ and $25^{\circ} \mathrm{C}$. Treatment conditions of free water or high RH and temperature were randomized among incubation chambers during repetitions of the various experiments. When required, light intensity within all chambers was adjusted to 180 $\mu \mathrm{mol} / \mathrm{m}^{2}$ per second at plant height and provided by incandescent and cool white T12 high output fluorescent bulbs. Plants in the low RH chamber were carefully pot-watered as required to avoid wetting the foliage.

Effect of temperature on in vitro germination of conidia. A conidial suspension of $1 \times 10^{6} / \mathrm{ml}$ was prepared and $0.5 \mathrm{ml}$ dispensed onto PDA in Petri dishes. The suspension was spread using a bent glass rod and then the dishes were sealed with Parafilm (Bemis Company, Inc., Neenah, WI) and incubated in darkness at 5, 10, 15, 20, 25, and $30^{\circ} \mathrm{C}$. After $3,9,13,15$, and $24 \mathrm{~h}$, the conidia were killed by flooding the agar surface with $95 \%$ ethanol and then the dishes were stored at $4^{\circ} \mathrm{C}$ until assessed. It was not possible to determine the percentage of germinated conidia because germ tubes grew from one or both ends of a conidium, and tube diameters often could not be distinguished from the conidium itself. Accordingly, the total length of a conidium and its germ tube(s) was measured on 20 arbitrary conidia per dish with a microscope. A single dish was assigned to each treatment and the experiment was conducted four times.

Effect of free water and RH on germination of conidia on leaves. An aqueous glycerol test was used to determine the effect of various levels of $\mathrm{RH}$ and free water on germination of $S$. vaccinii conidia on inoculated leaves. Petri dishes were used as incubation chambers in which the RH was maintained at $\sim 100,97.5,95,90$, or $85 \%$ with increasing concentrations of glycerol following the method of Forney and Brandl (1992). A filter paper was placed in an inverted lid of a dish to which $2.5 \mathrm{ml}$ of the appropriate glycerol solution was added and overlaid with a wire mesh shaped to rest $0.5 \mathrm{~cm}$ above the filter paper. Two blueberry plants were inoculated with a conidial suspension of $1 \times 10^{6} / \mathrm{ml}$. For the free water treatment, one random wet leaf from each inoculated plant was carefully detached and laid with the adaxial surface up on the wire mesh in a dish containing only water. The plants were then dried gently with fan-forced air (10 min) and one random dried leaf from each plant was also laid adaxial surface up on the mesh in dishes containing the appropriate glycerol solutions. The dishes were closed with the dish bottoms, sealed with Parafilm and wrapped with aluminum foil. The foil prevented condensation on the inner dish surfaces. An open dish with inoculated dried leaves was also incubated in a dark, high RH (>95\%) chamber described above to determine if conidia would germinate in a larger chamber for future whole plant experiments. All dishes were incubated at $25^{\circ} \mathrm{C}$ for $72 \mathrm{~h}$. Leaves from the free water treatment were then dried gently with fan-forced air and then all treatment leaves were placed on microscope slides. Conidia were killed and stained with a drop of $0.08 \%$ Trypan blue in $50 \%$ lactic acid. A coverslip was added, and the samples were stored at $4^{\circ} \mathrm{C}$ until assessed for the percentage of germinated conidia (100/leaf) with the aid of a microscope. The experiment was conducted four times.

Effect of free water and high RH on infection. Photo-entrained plants were randomly assigned to four treatments. Preliminary observations indicated that noninoculated, young blueberry leaves naturally develop a few small spots of unknown etiology almost identical to young lesions caused by $S$. vaccinii, but the spots did not increase in number or expand over time. Accordingly, noninoculated plants in the control treatment were incubated in the low $\mathrm{RH}(60 \% \mathrm{RH})$ growth chamber for the duration of the experiment to establish a base level of nondisease-related spotting as a reference for this and subsequent experiments. Additional plants were inoculated with $1 \times 10^{7}$ conidia/ml and those of the second and third treatments were immediately transferred to a wet chamber for 3 days. Plants of both treatments were then dried with fan-forced air, and plants of the second treatment were transferred to the low RH chamber. Plants of the third treatment were incubated for a further 3 days in a high $\mathrm{RH}$ chamber and then transferred to the low RH chamber. Plants of the 4th treatment were dried immediately after inoculation and incubated in a high RH chamber for 3 days and then transferred to the low RH chamber. All chambers operated at $25^{\circ} \mathrm{C}$ with a 14-h photoperiod. At 21 days postinoculation, disease 
severity was assessed as a measure of infection on the 10 most severely diseased leaves from each plant according to a 0 to 10 scale where $0=$ $0,1=0.1,2=0.2,3=0.4,4=0.8,5=1.6,6=3.2,7=6.4,8=12.8$, $9=25.6$, and $10=51.2 \%$ of the leaf area was covered with lesions as described in Ali et al. (2021). In perspective, scale ratings of 1 to 3 had 13, 26, and 52 leaf spots, respectively, whereas higher ratings had increasing numbers of spots with some touching other spots to reflect the natural coalescence of spots. There were three plants per treatment and the experiment was conducted four times.

Effect of light on infection. To prevent stomata from opening prematurely, plants were removed from the photo-entrainment chamber just before the beginning of the photoperiod, quickly inoculated in low light with $1 \times 10^{7}$ conidia/ml and then dried immediately with fan-forced air under dark conditions. Plants were then transferred to high RH chambers with random treatment assignments of: continuous darkness for 3 days; continuous darkness for 3 days followed by 4,8 , or $12 \mathrm{~h}$ of light; continuous light for 3 days, and 3 days with a 14-h photoperiod. All chambers were set at $25^{\circ} \mathrm{C}$. After incubation, two leaves from each plant were removed, stained, and stored as described and then plants were transferred to the low RH chamber (14-h photoperiod; $25^{\circ} \mathrm{C}$ ) and assessed for disease severity 21 days postinoculation as described. Germination percentage of the first 50 observed conidia/ leaf and percentage of penetration hyphae on 50 random germinated conidia was assessed. There were three plants per treatment and the experiment was conducted four times.

In addition to light microscopy observations, samples were also viewed with a Quanta 200 environmental scanning electron microscope (SEM; FEI Company, Hillsboro, OR). For surface observations, leaf samples from the 14-h photoperiod treatment were gently placed on a small piece of carbon conductive tape ( $9 \mathrm{~mm}$ in diameter) attached to a brass rectangular holder used in an Oxford CT 1500 cryotransfer system (Gatan, Abingdon, United Kingdom). Prepared samples were frozen in the cryo-prep chamber and sputter-coated with gold/palladium. Coated, frozen samples were then inserted into the environmental SEM and observed under high vacuum using a 10 to $15 \mathrm{kV}$ accelerating voltage and chamber temperature $>-160^{\circ} \mathrm{C}$. Images were captured digitally (grayscale with resolution at $1,024 \times$ 884 pixels).

Effect of temperature and duration of high RH on infection. Photo-entrained plants were inoculated with $1 \times 10^{7}$ conidia $/ \mathrm{ml}$ and dried immediately with fan-forced air. The plants were then randomly transferred to high $\mathrm{RH}$ chambers at $10,15,20,25$, or $30^{\circ} \mathrm{C}$ with 14 -h photoperiods for $2,3,4,5$, or 6 days. The plants were then transferred to the low RH chamber (14-h photoperiod; $25^{\circ} \mathrm{C}$ ) and disease severity on the 10 most diseased leaves per plant was assessed 21 days postinoculation. There were two plants per treatment and the experiment was conducted four times. A preliminary test showed that infection did not occur in the first $24 \mathrm{~h}$ of incubation at favorable temperatures of 20 or $25^{\circ} \mathrm{C}$ so this incubation time was not included in the experiment to conserve plant usage.

Effect of interrupted high RH on infection. Photo-entrained plants were inoculated with $1 \times 10^{7}$ conidia/ml and dried immediately with fan-forced air. The plants were then transferred to a high $\mathrm{RH}$ chamber for 2, 3, or 4 days or to alternating days in the high $\mathrm{RH}$ chamber and the low RH chamber for a total treatment time of 3 (high RH, low RH, high RH), 5, or 7 days. After treatment, the plants were transferred to the low RH chamber and disease severity on the 10 most diseased leaves per plant was assessed 21 days postinoculation. All chambers operated at $25^{\circ} \mathrm{C}$ with a 14-h photoperiod. There were three plants per treatment and the experiment was conducted four times.

Effect of plant age on infection. Blueberry plants with leaves 2 and 8 weeks old were photo-entrained and inoculated with $1 \times 10^{7}$ conidia/ml and dried immediately with fan-forced air. The plants were then transferred to a high RH chamber for 3 days and then to the low RH chamber. Disease severity on the 10 most diseased leaves per plant was assessed 21 days postinoculation. All chambers operated at $25^{\circ} \mathrm{C}$ with a $14-\mathrm{h}$ photoperiod. There were three plants per treatment and the experiment was conducted four times.
Data analysis. A series of individual experiments, each with its own set of objectives repeated four times, were conducted for each of the above seven aspects of the study. Five were single-factor experiments with a RCB design, and two of them were 2-way factorial experiments (i.e., Effect of Temperature on In Vitro Germination of Conidia; and Effect of Temperature and Duration of High RH on Infection). The random effects for the RCB design were "reps/subplot." For the two-way factorial experiments, a "split-plot design" was used, with random effects of replicates/main plot/subplot and fixed effects of temperature $\times$ time (incubation time [hours] or duration of high RH [days]). The temperature was randomized to the main plot and the hours or days randomized to the subplot. In each case, the time factor was the split and a polynomial contrast was completed on the time factor to evaluate the linear and quadratic response across time. Large residuals were removed before the final analysis. All data were subjected to a mixed model analysis using Genstat for Windows, 18th Ed. (VSN International, Hemel Hempstead, United Kingdom). After analysis, disease severity ratings were converted to percent of leaf area affected and are also presented to provide perspective.

\section{Results}

Effect of temperature on in vitro germination of conidia. Germination occurred mostly from either or both ends of conidia, and occasionally germ tubes formed laterally from intercalary cells of conidia. Branching of germ tubes was minimal during the 24-h observation period. Growth was initially slow, but increased after $13 \mathrm{~h}$ (Fig. 1). It was minimal at 5 and $10^{\circ} \mathrm{C}$, increased at 15 and $20^{\circ} \mathrm{C}$, was maximal at $25^{\circ} \mathrm{C}$, and decreased sharply at $30^{\circ} \mathrm{C}(P<0.001)$.

Effect of free water and $\mathrm{RH}$ on germination of conidia on leaves. Unlike the previous experiment where percentage of germination could not be determined, germinated conidia on leaves were clearly distinguishable after the 72 -h dark incubation period and were significantly affected by $\mathrm{RH}$ level $(P<0.001)$. In treatments where germination was pronounced, germ tubes were long, but showed minimal branching and grew along more or less straight paths. Only a few stomatal penetrations were observed. Conidia germination was $86.0,90.5$, and $81.3 \%$ in treatments of free water, 100 and $97.5 \% \mathrm{RH}$, respectively, whereas germination was only $28.3 \%$ at $95 \% \mathrm{RH}$ and $<3.0 \%$ at 90 and $85 \% \mathrm{RH}$ (Fig. 2). In these latter two treatments, conidia were swollen but germ tubes were very short and so germination percentage was difficult to assess accurately. Conidia germination in the Plexiglas humidity chamber $(>95 \% \mathrm{RH})$ was $70.5 \%$, indicating that a chamber with these conditions was suitable for further whole plant experimentation.

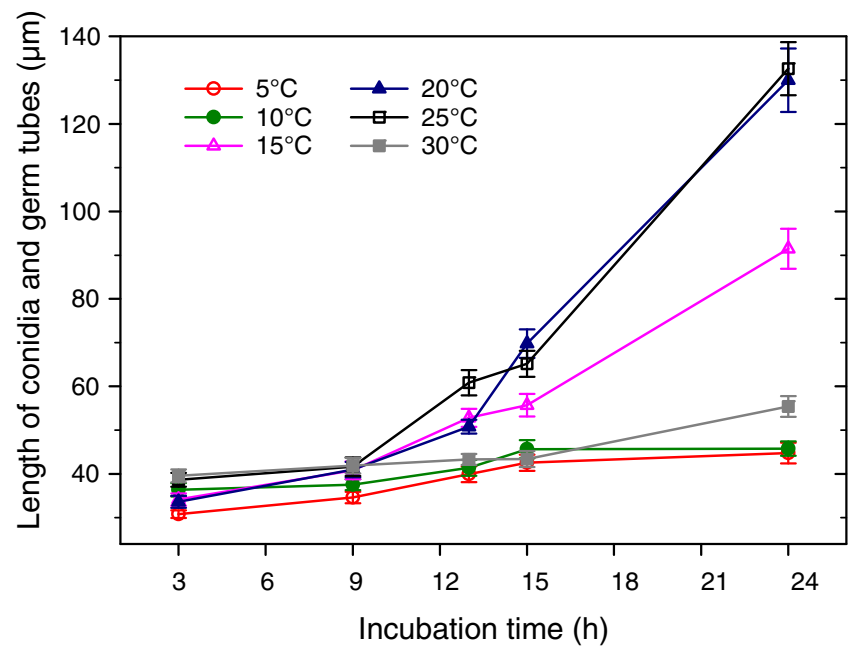

Fig. 1. Length of conidia and germ tubes of Sphaerulina vaccinii on PDA in relation to incubation temperature and time. Errors bars are standard error of the means $(P<0.001)$. 
Effect of free water and high RH on infection. Noninoculated control plants kept in the low RH chamber for the 21-day duration of the experiment showed slight natural spotting with an average severity rating of 0.9 (Fig. 3). Disease severity on inoculated plants incubated in a wet chamber for 3 days, dried and then transferred to the low RH chamber was low with a severity rating of only 2.0. However, disease severity on plants incubated in a wet chamber for 3 days, dried, and incubated in a high RH chamber for an additional 3 days and then transferred to the low RH chamber, or dried immediately after inoculation, incubated at high $\mathrm{RH}$ for 3 days and then transferred to the low $\mathrm{RH}$ chamber was much higher with mean severity ratings of 4.3 and 4.8 , respectively $(P<0.001)$. In this experiment and in subsequent ones, disease symptoms consistently began to develop $\sim 10$ days after inoculation.

Effect of light on infection. When plants were dried immediately after inoculation and then incubated in high RH chambers, the percentage of germinated conidia, percentage of stomatal penetrations, and subsequent disease severity, in general, were not affected by treatments of continuous darkness for 3 days, continuous darkness

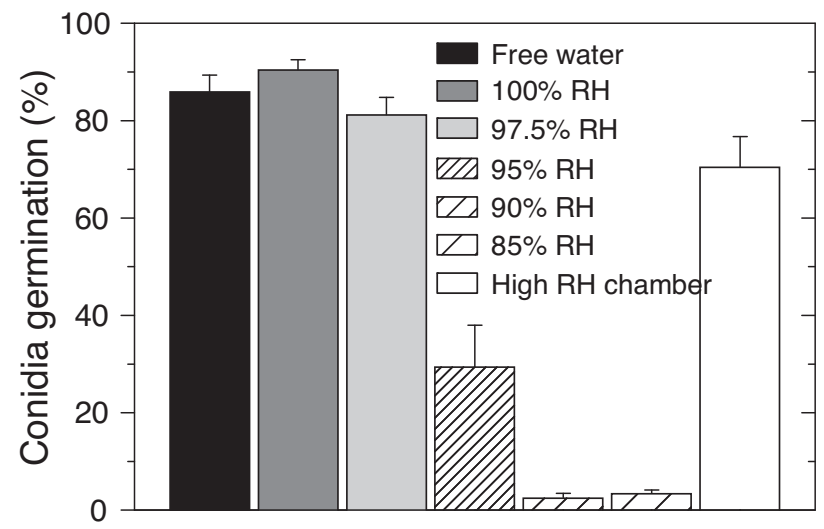

Fig. 2. Germination of conidia (\%) on the abaxial surface of detached blueberry leaves in relation to free water or various levels of $\mathrm{RH}$. Leaves were inoculated and incubated without drying or were fan-dried immediately after inoculation and then incubated at various levels of $\mathrm{RH}$ or in a high $\mathrm{RH}(>95 \%)$ plant incubation chamber. Leaves were incubated in darkness at $25^{\circ} \mathrm{C}$ for 3 days, stained, and then assessed for percentage of germinated conidia. Errors bars are standard error of the means $(P<0.001)$.

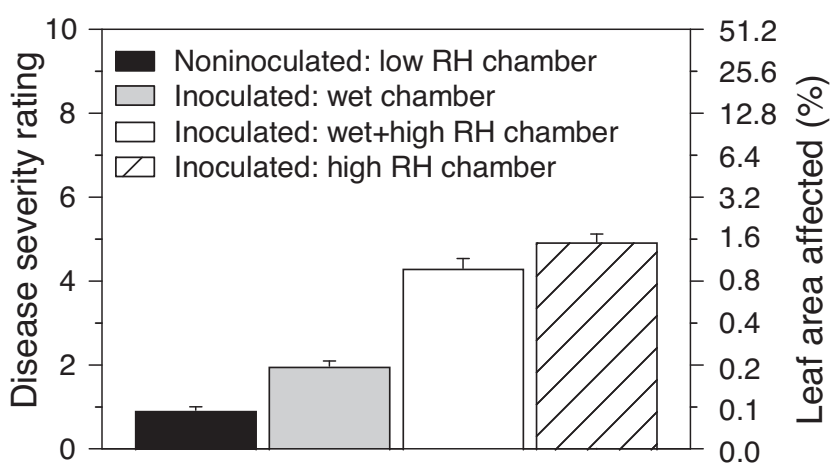

Fig. 3. Effect of free water and high $\mathrm{RH}$ on infection of blueberry leaves by Sphaerulina vaccinii. Noninoculated plants were maintained in a low RH $(60 \%)$ growth chamber to provide a base level of nondisease-related spotting that occurs naturally on blueberry leaves. Inoculated plants were incubated in a wet chamber for 3 days, fan-dried, and transferred to the low RH chamber, or incubated in a wet chamber for 3 days, fan-dried followed by 3 days in a high $\mathrm{RH}$ $(>95 \%)$ chamber, and then transferred to the low $\mathrm{RH}$ chamber, or fan-dried immediately after inoculation followed by 3 days in a high $\mathrm{RH}$ chamber and then transferred to the low RH chamber. Disease severity was assessed 21 days postinoculation on a 0 to 10 scale. All chambers operated at $25^{\circ} \mathrm{C}$ and a $14-\mathrm{h}$ photoperiod of $180 \mu \mathrm{mol} / \mathrm{m}^{2}$ per second. Error bars are standard error of the means $(P<0.001)$. for 3 days followed by 4,8 , or $12 \mathrm{~h}$ of light, or by continuous light for 3 days, although germination in continuous light was slightly higher (Table 1). However, plants exposed to a 14-h photoperiod showed a markedly higher percentage of germinated conidia $(P=0.002)$, stomatal penetrations $(P<0.001)$, and subsequent disease severity $(P<$ $0.001)$. Light microscope and SEM preparations showed that penetration of stomata was highly directional (Fig. 4A). When germ tubes grew in close proximity to a stomate, penetration hyphae developed on the germ tubes at $\sim 90^{\circ}$ angles and at the closest point to the stomate. The length of a penetration hypha from the main germ tube to the lip of a stomate was short and averaged $8.9 \mu \mathrm{m}$; the longest observed was $25.1 \mu \mathrm{m}(n=25)$. Germ tubes that grew over stomata and penetrated had very short penetration hyphae that were difficult to measure and so were not included in these measurements. Germ tubes sometimes penetrated stomata directly without first forming a penetration hypha. Penetrations into stomata were usually direct (Fig. 4B), but occasionally appressorium-like swellings formed over stomatal openings or disorganized hyphal matts developed as hyphae grew randomly within the stomatal pit above the sunken stomata. Penetrations into more than one stomate per single conidium occurred frequently as germ tubes grew and encountered additional stomata (Fig. 4A). When conidia were in close proximity to a stomate, short penetration hyphae formed on intercalary conidial cells occasionally with small appressorium-like swellings (Fig. 4C). Direct penetration into epidermal cells was not observed.

Effect of temperature and duration of high RH on infection. When plants were inoculated, dried immediately, and then incubated in high RH chambers at various temperatures, infection was significantly affected by temperature and duration of the postinoculation humid period $(P=0.046)$. A low level of infection occurred between 1 and 2 days at 20 and $25^{\circ} \mathrm{C}$ and continued to increase with increasing duration of high $\mathrm{RH}$ (Fig. 5). At $15^{\circ} \mathrm{C}$, infection occurred after 3 days of high $\mathrm{RH}$ and at $10^{\circ} \mathrm{C}$, infection began after 4 days of high $\mathrm{RH}$. Infection was minimal at $30^{\circ} \mathrm{C}$. When examined with light microscopy, leaves that had been incubated for 6 days showed an extensive branched hyphal network of epiphytic growth with many stomatal penetrations, especially at 20 and $25^{\circ} \mathrm{C}$. The amount of growth was substantially greater after 6 days than after 3 days as observed in the previous experiment.

Effect of interrupted high RH on infection. When plants were inoculated, dried immediately and then incubated in high RH chambers for 2, 3, or 4 days and then transferred to the low RH chamber, disease severity increased daily in a linear trend as in the previous experiment (Fig. 6). However, when plants were exposed to interruptions of high $\mathrm{RH}$ on alternate days, disease severity was significantly reduced $(P<0.001)$. On plants exposed to 1 day of high RH interrupted by 1 day of low RH followed by 1 day of high $\mathrm{RH}$, disease severity was reduced compared with two consecutive days of high RH. Disease severity increased slightly on plants exposed to 3 days of high $\mathrm{RH}$ interrupted by two alternate days of low $\mathrm{RH}$, but failed to increase further on plants exposed to 4 days of high $\mathrm{RH}$ interrupted by three alternate days of low $\mathrm{RH}$.

Effect of plant age on infection. The age of lowbush blueberry plants at the time of inoculation showed significant differences in susceptibility to infection $(P=0.038)$. Disease severity on 2-weekold plants was 6.6 in contrast to 2.4 on 8 -week-old plants, a 2.7-fold difference.

\section{Discussion}

Sphaerulina leaf spot and stem canker occurs annually in regions of commercial lowbush blueberry production, but environmental factors affecting infection and subsequent disease have not been previously studied. To the best of our knowledge, this is the first study investigating the relationships of temperature, $\mathrm{RH}$, light, and leaf age on infection and furthers our understanding of disease development.

Initial inoculum originates from pycnidia on overwintered leaves and in stem cankers. Pycnidia become mature at early bloom or slightly before and are mostly spent by the time all of the flowers have set, $\sim 3$ to 5 weeks later (Ali et al. 2021). During this period, 
rain is the agent for inoculum dispersal and depending on the intensity, conidia are splashed onto abaxial leaf surfaces. Although germination of conidia occurred in free water (Fig. 2) resulting in low levels of infection (Fig. 3), infection was strongly favored by high RH alone (Fig. 3). For optimal infection to occur in this scenario under field conditions, inoculum-laden splash droplets would have to first evaporate, but then canopy $\mathrm{RH}$ would have to remain above $95 \%$. This sequence likely occurs readily. By the time inoculum first becomes available during the flowering period, blueberry leaves are fully expanded creating a dense canopy that no doubt contributes to a high humidity environment, especially after a period of rain. In the maritime climate of Nova Scotia, rains are also often followed by prolonged periods of fog sometimes lasting for several days which also would provide high RH conditions ideal for infection. Casual observations in the field show that despite heavy fog and free water on the upper leaf surfaces, the lower surfaces are often dry to the touch, which would favor infection under these high RH conditions.

The interaction between temperature and duration of high $\mathrm{RH}$ also significantly affected infection by $S$. vaccinii. The optimum temperature for conidial germination and growth in vitro was $25^{\circ} \mathrm{C}$, but substantial growth did not occur before 13 to $15 \mathrm{~h}$ of incubation (Fig. 1). On blueberry leaves, infection did not occur in the first 24-h period after inoculation, but began during the second day at 20 and $25^{\circ} \mathrm{C}$. At 15 and $10^{\circ} \mathrm{C}$, infection did not occur until between the 3 rd and 4th and 4th and 5th days, respectively (Fig. 5). Thus, relatively prolonged periods of high $\mathrm{RH}$ are required for infection to occur. Under field conditions, springtime temperatures during rain events when inoculum is present are usually between 5 and $15^{\circ} \mathrm{C}$, but seldom reach 20 or $25^{\circ} \mathrm{C}$ and so high $\mathrm{RH}$ would have to persist for $>3$ days for significant infection to occur. However, after a period of rain, temperatures can sometimes increase substantially and if humidity within the plant canopy remains high, significant amounts of infection could occur. This study showed that disease severity increased daily with continued high $\mathrm{RH}$ during the infection period indicating that new infections were occurring daily from a single inoculation event (Fig. 5). When leaves were examined by light microscopy 6 days postinoculation, a network of branched hyphae with multiple stomatal penetrations was observed. However, the efficiency of these penetrations resulting in lesions is not known. Based on our observations, several-to-many penetrations may be required to form a lesion because there were many more penetrations than actual numbers of lesions that developed on diseased leaves. Casual observations of stained leaves from the field suggest that epiphytic growth by $S$. vaccinii can occur, but discerning growth of $S$. vaccinii was often obscured by growth of other colonists. Histopathology studies are required to further characterize epiphytic growth, hyphal ingress, and colonization in relation to disease development. Interestingly, a related pathogen Zymoseptoria tritici Crous (syn. Mycosphaerella graminicola; Mycosphaerellaceae, Dothideomycetes) that attacks wheat causing Septoria leaf blotch has similar requirements for infection. It too requires 24 to $48 \mathrm{~h}$ of moisture for infection and while infection can occur in free water (Chungu et al. 2001), it also occurs at high RH (Shaw 1991). This pathogen can also grow epiphytically on wheat leaves resulting in increased disease severity associated with increased periods of high humidity (Fones et al. 2017).

Under field conditions, alternating periods of high and low RH occur frequently, often on a daily basis that would have a significant impact on survivability of the pathogen. In the experiment where inoculated plants were exposed to 24-h alternating periods of high and low $\mathrm{RH}$, S. vaccinii was able to tolerate three dry periods, but not four (Fig. 6). Although this experiment tested only limited conditions, it does suggest that survival of conidia and/or epiphytic mycelium on leaves may last only a few days. For disease to progress by additional infections, a new infection period initiated by rain-dispersed conidia would be required. In the case of $Z$. tritici, interrupted humidity inhibited infection depending on the level of humidity and when it occurred during the light or dark phase of the photoperiod (Shaw 1991). Expanded studies on survival of $S$. vaccinii and epiphytic leaf colonization at various RH levels and durations are needed to further our understanding of infection and disease progress in the field.

Plant age was also a significant factor affecting infection and subsequent disease symptoms. Blueberry leaves were substantially more susceptible to infection when they were $\sim 2$ weeks old compared with those that had been fully expanded for $\sim 8$ weeks. Under field conditions, all leaves are usually fully expanded by the end of May with the oldest being $\sim 3$ to 4 weeks of age when inoculum first becomes available. This also coincides with the beginning of the flowering period and when this period is $>\sim 3$ to 5 weeks later, most of the available inoculum is depleted (Ali et al. 2021). Thus, the period of heightened susceptibility to infection is closely associated with the period of inoculum availability. Although not tested, these data also suggest that fungicides applied beginning at the early stages of inoculum maturity and heightened leaf susceptibility would be more efficacious than those applied near the end of the inoculum period. A similar close association between leaf age and susceptibility has also been observed with Septoria steviae Ishiba, Yokoyama \& Tani (Mycosphaerellaceae, Dothideomycetes) where 6-week-old stevia plants (Stevia rebaudiana Bertoni) were more susceptible to infection than plants just 3 weeks older (Reeleder 1999).

In addition to interactions of temperature and $\mathrm{RH}$, light also played a critical role in successful infection by $S$. vaccinii. Though some germination of conidia occurred in continuous darkness, few stomatal penetrations were observed and did not result in disease (Table 1). The addition of light for 4,8 , or $12 \mathrm{~h}$ after 3 days of continuous darkness did not improve germination or stomatal penetration. Germination was slightly higher in continuous light, but surprisingly, few stomatal penetrations occurred when stomata presumably would have been open for most of the time. However, under a normal field situation with a 14-h photoperiod, germination of conidia, penetration of stomata and subsequent disease severity were all markedly enhanced. Though photoperiodicity evidently is important for abundant germination and penetration of stomata, the mechanism(s) by which this

Table 1. Effect of light and darkness on germination of conidia and stomatal penetration by Sphaerulina vaccinii on lowbush blueberry leaves and subsequent disease severity

\begin{tabular}{lccc}
\hline Treatment $^{\mathbf{a}}$ & ${\text { Germination } \mathbf{( \% )}^{\mathbf{b}}}$ & ${\text { Penetration }(\boldsymbol{\%})^{\mathbf{c}}}$ & Disease severity $^{\mathbf{d}}$ \\
\hline 3 days continuous dark & 25.7 & 0.4 & $1.0(0.1)$ \\
3 days dark + 4-h light & 21.0 & 1.1 & $1.2(0.12)$ \\
3 days dark + 8-h light & 21.8 & 1.4 & $0.9(0.09)$ \\
3 days dark + 12-h light & 26.4 & 2.3 & $1.0(0.1)$ \\
3 days continuous light & 34.1 & 3.3 & $1.3(0.14)$ \\
3 days 14-h photoperiod & 51.6 & 19.6 & $6.3(5.4)$ \\
$P$ value & 0.002 & $<0.001$ & $<0.001$ \\
Scanning electron microscopy & 3.75 & 1.68 & 0.33 \\
\hline
\end{tabular}

${ }^{a}$ Plants were inoculated, fan-dried, and incubated in high RH $(>95 \%)$ chambers with various lighting regimes and durations and then transferred to a low RH (60\%) growth chamber until disease assessment at 21 days postinoculation. All chambers operated at $25^{\circ} \mathrm{C}$.

${ }^{b}$ After treatment, conidia were stained with $0.08 \%$ Trypan blue in $50 \%$ lactic acid. The first 50 conidia per leaf viewed microscopically were assessed for percentage of germination.

${ }^{\mathrm{c}}$ Fifty arbitrary germinated conidia per leaf were assessed for percentage of penetration into stomata.

${ }^{\mathrm{d}}$ Disease was assessed using a 0 to 10 rating scale. Values in parentheses are converted to percentage of diseased leaf area. 


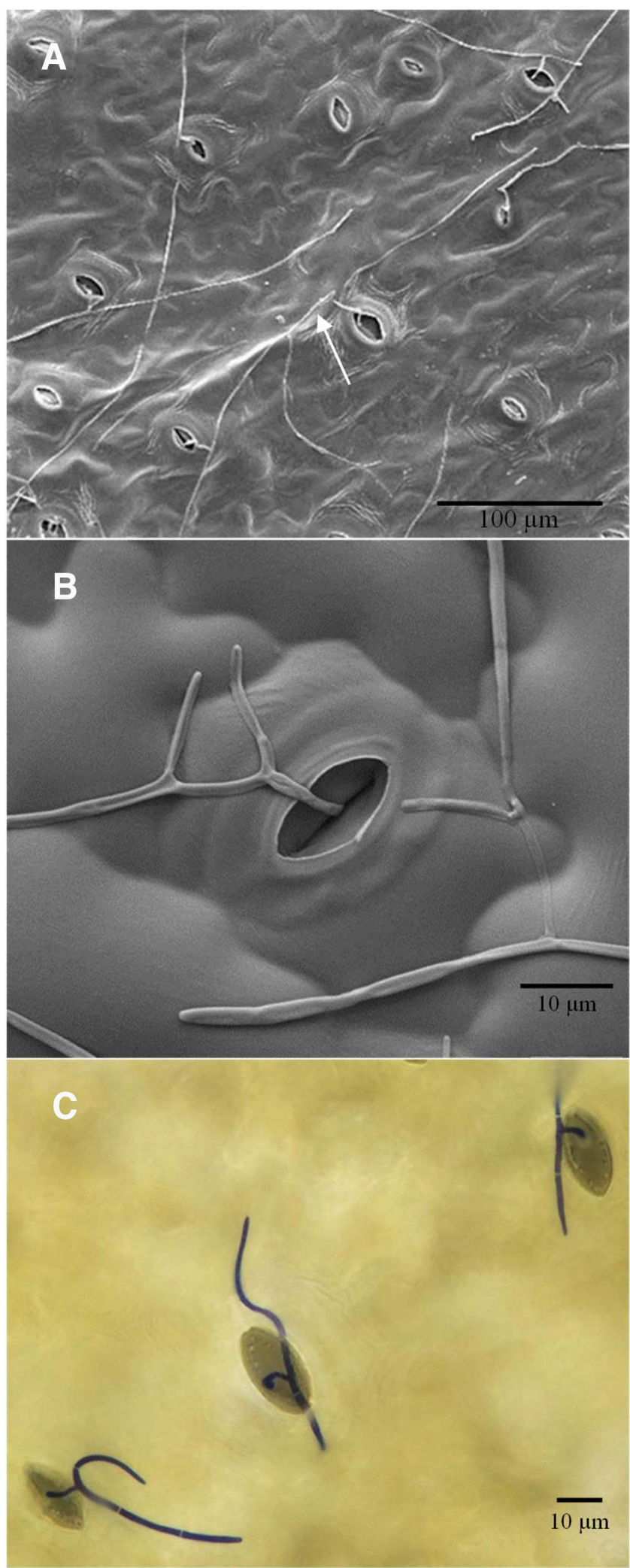

Fig. 4. Scanning electron and light microscope images of Sphaerulina vaccinii conidia germinating and penetrating the abaxial surface of blueberry leaves. Plants were inoculated, fan-dried, and then incubated in a high $\mathrm{RH}(>95 \%)$ chamber operating at $25^{\circ} \mathrm{C}$ with a 14 -h photoperiod of $180 \mu \mathrm{mol} / \mathrm{m}^{2}$ per second for 3 days. A, Stomatal penetration hyphae form at roughly $90^{\circ}$ angles on germ tubes when in close proximity to a stomate. Arrow indicates conidium from which a branched germ tube is penetrating two stomata. B, Direct penetration of hypha into a stomate. C, Short penetration hyphae with small appressorium-like swellings growing from intercalary cells of conidia. process occurs in $S$. vaccinii is not understood. It also is not known when and how quickly penetration hyphae form on germ tubes in relation to the photoperiod, though we suspect it begins shortly after stomata open in response to light. Casual observations showed that stomata opened $\sim 0.5 \mathrm{~h}$ after exposure to light in photo entrainment chambers, but we did not quantitatively follow stomatal opening in relation to onset and duration of the photoperiod in the various treatments. Further microscopy studies are required to quantify interactions of stomatal opening and formation of penetration hyphae over the course of photoperiods.

The orientation of penetration hyphae was also intriguing and indicated that a stomatal tropic response was at work. Penetration hyphae were almost always oriented at $90^{\circ}$ angles to the main germ tube or a branched hypha and took a more or less straight and shortest path to the stomate (Fig. 4). Though speculative, this tropism might be chemotropic. In the case of Cercospora zeae-maydis Tehon \& Daniels (Mycosphaerellaceae, Dothideomycetes), the causal agent of gray leaf spot of maize, stomatal tropism, and appressorium formation were impaired when atmospheric oxygen levels were altered and when the fungus was exposed to an increasing atmospheric oxygen gradient in vitro, appressorium-like structures were formed

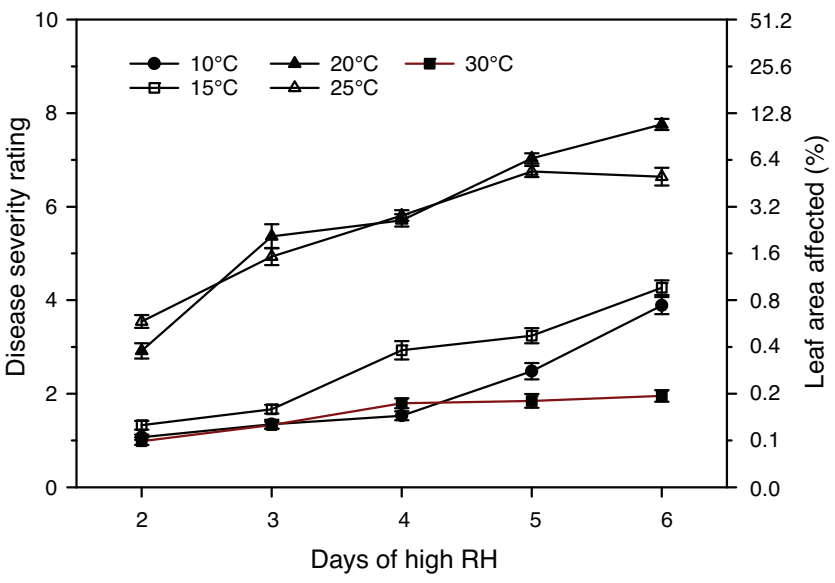

Fig. 5. Effect of temperature and duration of high $\mathrm{RH}$ on infection of blueberry leaves by Sphaerulina vaccinii. Plants were inoculated, fan-dried, and incubated in high $\mathrm{RH}(>95 \%)$ chambers at various temperatures for various durations. The plants were then transferred to a low $\mathrm{RH}(60 \%)$ growth chamber $\left(25^{\circ} \mathrm{C}\right)$ until disease assessment at 21 days postinoculation. Disease severity was assessed on a 0 to 10 scale. All chambers operated with a 14-h photoperiod of $180 \mu \mathrm{mol} / \mathrm{m}^{2}$ per second. Errors bars are standard error of the means $(P=0.046)$.

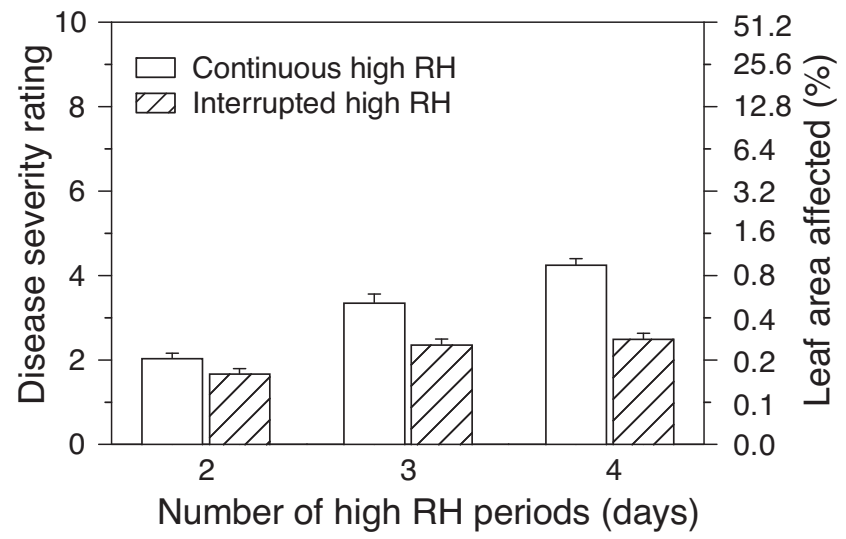

Fig. 6. Effect of interrupted high RH on infection of blueberry leaves by Sphaerulina vaccinii. Plants were inoculated, fan-dried, and incubated in high $\mathrm{RH}(>95 \%)$ chambers for 2, 3, or 4 days or to alternating days of high $\mathrm{RH}$ and low $\mathrm{RH}(60 \%)$. Plants were then transferred to a low RH $(60 \%)$ growth chamber until disease assessment at 21 days postinoculation. Disease severity was assessed on a 0 to 10 scale. All chambers operated at $25^{\circ} \mathrm{C}$ with a 14 -h photoperiod of $180 \mu \mathrm{mol} / \mathrm{m}^{2}$ per second. Errors bars are standard error of the means $(P=0.004)$ 
demonstrating a chemotropic response (Hirsch 2014). A similar tropic response may be at work in $S$. vaccinii. When free water was maintained on inoculated leaves during a 14-h photoperiod (Fig. 3), infection and subsequent disease severity were low due possibly to an impaired ability of the fungus to sense an oxygen gradient in the water film. However, when plants were dried immediately after inoculation and exposed to high RH alone, infection occurred at high levels (Fig. 3).

This study has focused on leaf infections, but stem cankers caused by $S$. vaccinii are also an important factor affecting yields. Interestingly, given the high density of lesions occurring on leaves, one might expect there to be many lesions on stems as well, but there are usually only a few by comparison (Ali et al. 2021). The reason for this difference is not known, but perhaps is related to a lower stomatal density on stems, an aspect requiring more study.

In summary, this study has shown that infection of lowbush blueberry by $S$. vaccinii is a complex process requiring prolonged periods of high RH after a rain event. It is unlikely that a simple weatherbased model can be developed at this point to assist in timing of fungicide sprays based only on relationships of duration of high $\mathrm{RH}$ and temperature because infection is also strongly affected by interaction with the photoperiod and cycles of drying. More research is required to elucidate environmental conditions affecting survival of conidia after leaf deposition, survival of epiphytic mycelium, and the timing of infections during the photoperiod.

\section{Acknowledgments}

Technical help of Kentville Research and Development Centre greenhouse staff and summer/co-op students is gratefully acknowledged. The authors thank S. A. E. Fillmore (regional statistician), Agriculture and Agri-Food Canada, Kentville, Nova Scotia for assisting with data analysis.

\section{Literature Cited}

Ali, S., Hildebrand, P. D., Renderos, W. E., and Abbasi, P. A. 2021. Identification and characterization of Sphaerulina vaccinii as the cause of leaf spot and stem canker in lowbush blueberry and its epidemiology. Phytopathology 111:1560-1570.

Annis, S. L., and Stubbs, C. S. 2004. Stem and leaf diseases and their effects on yield in Maine lowbush blueberry fields. Small Fruits Rev. 3:159-167.

Bell, D. J., Rowland, L. J., Stommel, J., and Drummond, F. A. 2010. Yield variation among clones of lowbush blueberry as a function of genetic similarity and self-compatibility. J. Am. Soc. Hortic. Sci. 135:259-270.

Chungu, C., Gilbert, J., and Townley-Smith, F. 2001. Septoria tritici blotch development as affected by temperature, duration of leaf wetness, inoculum concentration, and host. Plant Dis. 85:430-435.

Demaree, J. B., and Wilcox, M. S. 1947. Fungi pathogenic to blueberries in the eastern United States. Phytopathology 37:487-506.

De Pascual-Teresa, S., Moreno, D. A., and García-Viguera, C. 2010. Flavanols and anthocyanins in cardiovascular health: a review of current evidence. Int. J. Mol. Sci. 11:1679-1703.

Drummond, F., Annis, S., Smagula, J. M., and Yarborough, D. E. 2009. Organic production of wild blueberries. I. Insects and disease. Acta Hortic. 810:275-286.

Fones, H. N., Eyles, C. J., Kay, W., Cowper, J., and Gurr, S. J. 2017. A role for random, humidity-dependent epiphytic growth prior to invasion of wheat by Zymoseptoria tritici. Fungal Genet. Biol. 106:51-60.
Forney, C. F., and Brandl, D. G. 1992. Control of humidity in small controlledenvironment chambers using glycerol-water solutions. HortTechnology 2: $52-54$.

Forney, C. F., and Kalt, W. 2011. Blueberries and cranberries. Pages 51-73 in: Health Promoting Properties of Fruits and Vegetables. L. Terry, ed. CABI, Wallingford, UK

Hildebrand, P. D., and Braun, P. G. 1991. Factors affecting infection of lowbush blueberry by ascospores of Monilinia vaccinii-corymbosi. Can. J. Plant Pathol. 13:232-240.

Hildebrand, P. D., and Renderos, W. E. 2017. Septoria leaf spot and stem canker. Pages 54-57 in: Compendium of Blueberry, Cranberry, and Lingonberry Diseases and Pests, 2nd Ed. J. J. Polaschock, F. L. Caruso, A. L. Averill, and A. C. Schilder, eds. The American Phytopathological Society, St. Paul, MN.

Hildebrand, P. D., Renderos, W. E., and Delbridge, R. W. 2016. Diseases of Lowbush Blueberry and Their Identification. Agriculture and Agri-Food Canada, Kentville, NS, Canada.

Hildebrand, P. D., Renderos, W. E., and Fillmore, S. A. E. 2010a. Severity of Septoria leaf spot and stem canker and leaf rust in lowbush blueberry fields pruned by mowing or burning. Can. Plant Dis. Surv. 90:155-157.

Hildebrand, P. D., Renderos, W. E., Fillmore, S. A. E., and Walker, B. 2010 b. Symptoms and epidemiology of a Septoria spp. causing leaf and stem canker of lowbush blueberry. Can. J. Plant Pathol. 32:416.

Hirsch, R. L. 2014. Dissection of gray leaf spot of maize through functional genomics. In: Graduate Theses and Dissertations. 2091. University of Arkansas, Fayetteville, AR. https://scholarworks.uark.edu/etd/2091

Ojiambo, P. S., and Scherm, H. 2005. Survival analysis of time to abscission of blueberry leaves affected by Septoria leaf spot. Phytopathology 95: 108-113.

Ojiambo, P. S., Scherm, H., and Brannen, P. M. 2006. Septoria leaf spot reduces flower bud set and yield potential of rabbiteye and southern highbush blueberries. Plant Dis. 90:51-57.

Percival, D. C., and Dawson, J. K. 2009. Foliar disease impact and possible control strategies in wild blueberry production. Acta Hortic. 810:345-354.

Reeleder, R. D. 1999. Septoria leaf spot of Stevia rebaudiana in Canada and methods for screening for resistance. J. Phytopathol. 147:605-613.

Roloff, I., Scherm, H., and van Iersel, M. W. 2004. Photosynthesis of blueberry leaves as affected by Septoria leaf spot and abiotic leaf damage. Plant Dis. $88: 397-401$.

Scherm, H. 2017. Septoria leaf spot. Pages 41-43 in: Compendium of Blueberry, Cranberry, and Lingonberry Diseases and Pests, 2nd Ed. J. J. Polaschock, F. L. Caruso, A. L. Averill, and A. C. Schilder, eds. The American Phytopathological Society, St. Paul, MN.

Scherm, H., Brannen, P. M., Ojiambo, P. S., Savelle, A. T., Krewer, G., and Bruorton, M. D. 2003. Blueberry leaf spots: epidemiology, yield losses, and control. Pages 57-66 in: Proceedings of the Southeast Blueberry Conference, Savannah, GA.

Scherm, H., and Krewer, G. 2003. Blueberry production in Georgia: historical overview and recent trends. Small Fruits Rev. 2:83-91.

Scherm, H., and Krewer, G. 2008. Disease management in organic rabbiteye blueberries. Int. J. Fruit Sci. 8:69-80.

Shaw, M. W. 1991. Interacting effects of interrupted humid periods and light on infection of wheat leaves by Mycosphaerella graminicola (Septoria tritici). Plant Pathol. 40:595-607.

Vander Kloet, S. P. 1978. Systematics, distribution, and nomenclature of the polymorphic Vaccinium angustifolium. Rhodora 80:358-371.

Williamson, J. G., and Miller, E. P. 2002. Early and mid-fall defoliation reduces flower bud number and yield of southern highbush blueberry. HortTechnology 12:214-216.

Yarborough, D., Drummond, F., Annis, S., and D'Appollonio, J. 2017. Maine wild blueberry systems analysis. Acta Hortic. 1180:151-160. 